\title{
Contexto de Formação e Sofrimento Psíquico de Estudantes de Medicina
}

\author{
The training context and mental suffering \\ among student doctors
}

\author{
João Brainer Clares de Andrade \\ José Jackson Coelho Sampaio \\ Lara Maciel de Farias ${ }^{I I}$ \\ Lucas da Ponte Melo ${ }^{I I I}$ \\ Dalmy Pinheiro de Sousa ${ }^{I V}$ \\ Ana Luisa Barbosa de Mendonça ${ }^{I V}$ \\ Francisco Felinto Aguiar de Moura Filho ${ }^{I V}$ \\ Ingrid Sorensen Marinho Cidrãov
}

\section{PALAVRAS-CHAVE}

- Estudantes de Medicina;

- Saúde Mental;

- Educação Médica.

\section{KEYWORDS}

- Student Doctors;

- Mental Health;

- Medical Training.

Recebido em: 15/06/2013

Reencaminhado em: 22/08/2013

Reencaminhado em: 30/12/2013

Aprovado em: 16/01/2014

REVISTA BRASILEIRA DE EDUCAÇÃO MÉDICA
I Universidade Estadual do Ceará, Ceará, CE, Brasil.

"I Estácio de Juazeiro do Norte, Juazeiro do Norte, CE, Brasil.

III Universidade Federal do Ceará, Ceará, CE, Brasil.

IV Universidade de Fortaleza, Fortaleza, CE, Brasil.

${ }^{v}$ Centro Universitário UniChristus, Fortaleza, Ceará, CE. Brasil.

\section{RESUMO}

A formação em Medicina é densa e com grandes responsabilidades. Contato com a morte, ambiente competitivo, privação de lazer e sensação de insegurança técnica funcionam como um retrato da formação. Objetiva-se descrever os diferentes processos que interferem no sofrimento psíquico discente em todas as escolas médicas do Ceará. O estudo acompanhou, do segundo ao sexto ano, 40 alunos da Universidade Estadual do Ceará (Uece) e 20\% dos demais estudantes com ingresso comum em todas as outras escolas cearenses. Utilizou-se o Self-Report Questionnaire-20 para avaliar transtornos mentais leves (TML). A maior prevalência de suspeitos de portar TML foi de 53,3\% na Uece, com 20\% dos alunos procurando ajuda psicológica. Nas outras escolas, 48,5\% foram suspeitos e 18,2\% procuraram ajuda. Depressão, insônia, problema pessoal, privação de lazer e insegurança técnica atuaram sobre o sofrimento. A formação médica representa um período de dúvidas, receios e tensões. Os currículos precisam considerar como os estudantes lidam com a formação. As escolas devem tornar seus serviços de apoio mais integrados à execução dos currículos, focando os dois últimos anos, oferecendo suporte às tensões pessoais e familiares.

\begin{abstract}
Medical training is an intense process that brings considerable responsibilities. Contact with death, a competitive environment, deprivation of leisure and a sense of technical insecurity are typical characteristics of the student's experience. This study aims to describe the different processes that affect psychological distress among students at all the medical schools in Ceará state. The study followed 40 second to sixth year students from the State University of Ceará (UECE) and 20\% of the other students admitted by regular means to the other schools in Ceará. The Self-Report Questionnaire-20 was used to evaluate slight mental disorders (SMD). Suspected cases of SMD were most prevalent at UECE, 53.3\% of the students, and $20 \%$ had sought psychological help. In the other schools, there were $48.5 \%$ suspected cases, and $18.2 \%$ who had sought help. Psychological suffering was found to be engaged by depression, insomnia, personal problems, deprivation of leisure and technical insecurity. Medical education is a period of doubts, fears and tensions. The curricula need to consider how students deal with their own training. Schools should improve the integration between their support services and the implementation of the curricula, focusing on the last two years of study, offering support for personal and family tensions.
\end{abstract}

ABSTRACT 


\section{INTRODUÇÃO}

As exigências da organização social são fundantes, estruturantes, geradoras das opções, dentro das molduras normativas, para as escolhas profissionais ao término da adolescência. A graduação em Medicina, a exemplo, constitui uma escolha que exigirá afeição pela labuta médica, e, sobretudo, preparação contínua para atingir a concorrência crescente e o nível intelectual dos demais atores da mesma escolha ${ }^{1}$.

Os diferentes cursos de Medicina apresentam grande interesse na aprendizagem de seus alunos, gerando um aprimoramento nas formas de intervenção, além de atualização em relação a novas técnicas e melhoria das equipes profissionais, mas essa mesma inquietação não se evidencia em relação à saúde mental de seu educando². $\mathrm{O}$ resultado é a formação de um estudante que, nos semestres finais do curso, tem conhecimentos sobre as diferentes áreas biomédicas, mas apresenta deficiências no relacionamento com o ser humano, embora a eficácia de sua abordagem dependa, também, desse fator ${ }^{3}$.

A concepção de estudos comunitários, por visões históricas baseadas nas ciências sociais, na psicologia e na clínica médica, é utilizada hoje para delinear estratégias e traçar metas em saúde pública, o que contempla essa atuação de forma muito mais integrada e abrangente. Com isso, o contexto de formação em Medicina torna-se ainda mais denso, aglutinando responsabilidades sociais e técnicas desde o primeiro ano letivo. O contato precoce com a morte, a personalização do cadáver nas aulas práticas, o ambiente acirrado vindo dos cursinhos pré-vestibulares, a demanda dos professores, a exigência pela excelência em avaliações como uma forma de perpetuar o perfil do Ensino Médio, a proximidade com a realidade do paciente, o sofrimento pessoal e familiar, a privação de lazer, a carga horária extenuante, as incertezas quanto ao exercício da profissão, o contato com preceptores, residentes e alunos de outras faculdades como modelo de concorrência, a sensação de insegurança técnica e as incertezas quanto ao mercado de trabalho funcionam como um retrato do contexto de formação em Medicina hoje i,2,3,4. $^{1,2}$

O período de maior sofrimento psíquico vivenciado pelos estudantes corresponde àquele em que entram em contato com pacientes gravemente enfermos; em que a partir do quarto semestre, além das exigências acadêmicas, os alunos começam a sua formação clínica, que é um novo e importante fator estressante, havendo um aumento significativo de sintomas depressivos também no quarto ano, o que provavelmente se deve ao fato de que os alunos precisam fazer escolhas quanto ao seu internato ${ }^{5,6}$.
No Ceará, o panorama da formação médica é definido por sete centros formadores. Em Fortaleza: Universidade Estadual do Ceará, Universidade Federal do Ceará, Universidade de Fortaleza e o Centro Universitário UniChristus. No interior do Estado, contemplando duas macrorregiões, há os dois campi da Universidade Federal do Ceará e a Faculdade de Medicina Estácio de Juazeiro do Norte. Nesse contexto, entre os sete centros formadores de médicos, há uma distribuição de vagas de forma a graduar 652 novos médicos anualmente, entre formandos de faculdades particulares (332) e públicas (320), tendo por base as vagas oferecidas em 2009.

Sobre a perspectiva da formação médica, é preciso reconhecer as novas demandas sociais. A partir de tais demandas, torna-se possível compreender as prerrogativas utilizadas pelas escolas médicas na formação do futuro médico e, portanto, sua ambiência de condutas e habilidades ${ }^{7,8}$. A realidade sanitária atual exige dos aparelhos formadores um esforço adicional no processo de capacitação dos recursos humanos, tendo em vista a relativamente escassa disponibilidade de profissionais com formação generalista e vocacionados às políticas públicas de saúde?.

Vários estudos relatam que a relação professor-aluno, atuação do corpo docente, qualidade de ensino, fragmentação do saber, prática distante da teoria, metodologia de ensino-aprendizagem, estrutura curricular e infraestrutura são fatores possíveis de constituírem problemas durante a formação do estudante de Medicina ${ }^{10,11,12}$.

A quantidade e a gravidade de estresse vivido por estudantes de Medicina podem variar de acordo com as definições da escola médica, do currículo, da avaliação do sistema, etc. Estudos prévios realizados em escolas médicas em diferentes países têm relatado diferentes níveis de estresse associados a tais características pedagógicas ${ }^{13}$.

Sobre as principais demandas que levam o estudante e procurar ajuda psicológica, destacam-se as dificuldades de relacionamento amoroso (mais presentes entre os alunos quando dizem respeito especialmente à vida sexual) e dificuldade de relacionamento familiar (mais presente entre os alunos quando envolve conflitos com o papel de autoridade dos pais e mais presente entre as alunas quando diz respeito às dificuldades de separação e distância da família) ${ }^{2}$.

É objetivo geral descrever os diversos processos psicossociais, acadêmicos, pessoais e familiares que possam interferir positiva ou negativamente sobre o sofrimento psíquico no processo de formação de estudantes de Medicina em todas as escolas médicas do Ceará, Brasil, em atividade autorizada no período de 2009 a 2013, integrando os dados às 
perspectivas e modelos de formação presentes nos projetos político-pedagógicos dos cursos de Medicina do Ceará, em abordagem exploratória, e descrição de fatores que interferem no pensamento de desistência do curso, procura por auxílio psicológico e mau desempenho acadêmico dos futuros médicos.

\section{TRATAMENTO METODOLÓGICO}

Os dados foram obtidos por meio de três distintos formulários, tendo por base as características socioculturais, pessoais e acadêmicas que direta ou indiretamente estão associadas à formação e ao sofrimento mental de estudantes de Medicina, a partir de revisão de literatura.

O desenho geral da pesquisa é uma coorte com grupo a partir do terceiro semestre acadêmico, no mês de maio de 2009. A escolha de alunos do terceiro semestre/período é baseada no amadurecimento progressivo obtido pelos estudantes de Medicina com a adaptação esperada com o curso universitário.

O grupo de estudantes selecionado como referencial do estudo foi a sexta turma do curso de Medicina da Universidade Estadual do Ceará (Uece), com 40 alunos regularmente matriculados em janeiro de 2009. Para a análise comparativa, utilizou-se uma amostragem aleatória, de 20\% dos alunos regularmente matriculados e que estavam cursando, em maio de 2009, também o terceiro semestre acadêmico de todas as outras escolas médicas cearenses. A taxa de perda de participantes no último ano foi de 34,3\%, em comparação ao grupo de partida. As perdas se deram por óbito (1), reprovações (3) e recusas no preenchimento dos dados.

Em todas as coletas anuais, houve a aplicação do Self-Report Questionnaire (SRQ-20), sendo um grupo de 20 questões com respostas sim ou não, um formulário reconhecido pela Organização Mundial de Saúde (OMS) e validado no Brasil $^{14}$. O valor de corte necessário para enquadrar o aluno pesquisado como suspeito de ser portador de sofrimento mental é igual ou maior que seis respostas afirmativas para homens e oito ou mais respostas afirmativas para mulher. Esse formulário atende ao objetivo de identificar o grupo suspeito de portar e o não suspeito de portar transtorno mental leve.

Nas duas últimas aplicações, compatíveis com os grupos em curso do internato médico, a aplicação dos formulários deu-se por modelo eletrônico construído na plataforma FormStack (C). O formulário virtual foi enviado por e-mail aos alunos participantes por meio de cadastro que realizaram junto aos colaboradores nas respectivas escolas médicas. $\mathrm{O}$ formulário virtual é isento de qualquer recurso que possa identificar o aluno, exceto pelos campos de idade, sexo e escola médica.

A pesquisa é de natureza quantitativa, com perspectiva qualitativa, por meio de análise longitudinal de números absolutos e percentuais e medidas de tendência central. Houve aplicação de testes estatísticos para verificar possíveis associações entre variáveis, com software de estatística Epi Info 3.5.2 para construção de um banco de dados que facilite a interpretação dos resultados obtidos, por meio de regressão logística, que considerou todas as variáveis relacionadas ao desfecho e depois gerou novo modelo com as variáveis que pontuaram $p$-value inferior ou igual a 0,2 . Os resultados com $p$-value menor que 0,05 foram considerados de significado estatístico.

A pesquisa encontra-se adequada à resolução $\mathrm{CNS}$ 196/96, com aceite livre e esclarecido dos entrevistados, garantia de anonimato e aprovação no Comitê de Ética em Pesquisa da Universidade Estadual do Ceará.

\section{RESULTADOS}

A população e amostra estudadas tiveram média de idade de 21,5 anos na Uece e 21 anos nas outras escolas (OE). A distribuição por sexo foi de $51,5 \%$ de mulheres na Uece e $45 \%$ nas demais escolas.

O modelo de formação encontrado nas escolas médicas $^{15-19}$ cearenses é congruente com o estipulado pelas Diretrizes Curriculares Nacionais para Graduação em Medicina (2001). Há o foco na formação generalista, com ampla competência comunitária, com a presença de um eixo humanístico. Somente no curso de Medicina da Uece foi observada a competência de o médico zelar pela própria saúde física e mental, de forma objetiva, além da presença, na disciplina de Clínica Médica II, do tópico "Qualidade de Vida"15. No entanto, o projeto da Uece não cita serviços da própria universidade para o apoio psicológico ao estudante de Medicina. A presença ou menção a serviço de apoio psicopedagógico foi encontrada em todas as demais escolas médicas cearenses, exceto na Universidade Federal do Ceará, campus Cariri.

A Tabela 1 sumariza os elementos psicossociais, pessoais e familiares apontados pelos alunos. A percepção de mudança de humor foi apontada por mais de $60 \%$ dos alunos nos dois grupos no primeiro ano do curso. A dificuldade com novas amizades foi pior no grupo da Uece no primeiro ano de internato. Nas demais escolas, o pico (30\%) se deu no último ano da graduação, após ter decrescido no quarto e quinto anos (5\% e $6,9 \%$, respectivamente). 
Tabela 1

Distribuição de prevalências de acordo com o ano acadêmico estudado.

Aspectos psicossociais

\begin{tabular}{|c|c|c|c|c|c|}
\hline Variável & $2^{\circ}$ ano & $3^{\circ}$ ano & $4^{\circ}$ ano & $5^{\circ}$ ano & $6^{\circ}$ ano \\
\hline Idade (média) & $\begin{array}{l}\text { Uece } \\
21,5 \text { anos } \\
\text { OE } \\
21 \text { anos }\end{array}$ & - & - & - & - \\
\hline Distribuição por sexo & $\begin{array}{l}\text { Uece - Mulheres } \\
-52,5 \% \\
\text { OE -Mulheres- } \\
45 \%\end{array}$ & - & - & - & - \\
\hline Percebeu mudança de humor & $\begin{array}{l}\text { Uece }-62,5 \% \\
\text { OE }-75,3 \%\end{array}$ & - & - & - & - \\
\hline Dificuldade com novas amizades & $\begin{array}{l}\text { Uece }-10 \% \\
\text { OE - } 21,5 \%\end{array}$ & $\begin{array}{l}\text { Uece }-12,5 \% \\
\text { OE - } 20 \%\end{array}$ & $\begin{array}{l}\text { Uece }-15 \% \\
\text { OE }-5 \%\end{array}$ & $\begin{array}{l}\text { Uece }-20 \% \\
\text { OE }-6,9 \%\end{array}$ & $\begin{array}{l}\text { Uece }-12,5 \% \\
\text { OE }-30 \%\end{array}$ \\
\hline Apresentou quadro depressivo & $\begin{array}{l}\text { Uece }-25,6 \% \\
\text { OE - } 15,4 \%\end{array}$ & - & - & - & - \\
\hline Problema pessoal ou familiar & $\begin{array}{l}\text { Uece - 35\% } \\
\text { OE - 33,8\% }\end{array}$ & $\begin{array}{l}\text { Uece }-35 \% \\
\text { OE }-32,3 \%\end{array}$ & $\begin{array}{l}\text { Uece }-32,5 \% \\
\text { OE }-25 \%\end{array}$ & $\begin{array}{l}\text { Uece }-46,6 \% \\
\text { OE }-27,6 \%\end{array}$ & $\begin{array}{l}\text { Uece }-58,1 \% \\
\text { OE }-39,4 \%\end{array}$ \\
\hline Não está adaptado à cidade & - & $\begin{array}{l}\text { Uece }-5 \% \\
\text { OE }-12,3 \%\end{array}$ & $\begin{array}{l}\text { Uece }-2,5 \% \\
\text { OE }-3,3 \%\end{array}$ & $\begin{array}{l}\text { Uece }-10,3 \% \\
\text { OE }-6,9 \%\end{array}$ & $\begin{array}{l}\text { Uece }-3,1 \% \\
\text { OE }-15,5 \%\end{array}$ \\
\hline Mora sem os pais & - & $\begin{array}{l}\text { Uece }-30 \% \\
\mathrm{OE}-37 \%\end{array}$ & $\begin{array}{l}\text { Uece }-25 \% \\
\text { OE }-41,7 \%\end{array}$ & - & - \\
\hline Passou a consumir álcool & $\begin{array}{l}\text { Uece }-20 \% \\
\text { OE }-47,7 \%\end{array}$ & - & - & - & - \\
\hline Consome álcool & - & $\begin{array}{l}\text { Uece- } 35 \% \\
\text { OE - } 64,6 \%\end{array}$ & $\begin{array}{l}\text { Uece- } 47,5 \% \\
\text { OE- } 76,7 \%\end{array}$ & $\begin{array}{l}\text { Uece- } 60 \% \\
\text { OE - } 58,6 \%\end{array}$ & $\begin{array}{l}\text { Uece- } 62,2 \% \\
\text { OE- } 69,7 \%\end{array}$ \\
\hline Consome alguma droga psicoativa & - & $\begin{array}{l}\text { Uece- } 7,5 \% \\
\text { OE - } 10,8 \%\end{array}$ & $\begin{array}{l}\text { Uece- } 2,6 \% \\
\text { OE - } 1,7 \%\end{array}$ & $\begin{array}{l}\text { Uece- } 3,3 \% \\
\text { OE }-3,4 \%\end{array}$ & $\begin{array}{l}\text { Uece- } 6,3 \% \\
\text { OE - } \\
9 \%\end{array}$ \\
\hline Parou de realizar atividade física & $\begin{array}{l}\text { Uece }-55 \% \\
\text { OE }-44,6 \%\end{array}$ & - & - & - & - \\
\hline Realiza atividade física regular & - & $\begin{array}{l}\text { Uece- } 35 \% \\
\text { OE - } 37 \%\end{array}$ & $\begin{array}{l}\text { Uece- } 37,5 \% \\
\text { OE - } 43,3 \%\end{array}$ & $\begin{array}{l}\text { Uece- } 33 \% \\
\text { OE - } 24 \%\end{array}$ & $\begin{array}{l}\text { Uece- } 34,4 \% \\
\text { OE - } 39,4 \%\end{array}$ \\
\hline Privou-se de alguma atividade de lazer & $\begin{array}{l}\text { Uece }-72,5 \% \\
\text { OE - } 61,5 \%\end{array}$ & $\begin{array}{l}\text { Uece- } 72,5 \% \\
\text { OE - } 67,7 \%\end{array}$ & $\begin{array}{l}\text { Uece- } 60 \% \\
\text { OE - } 66,7 \%\end{array}$ & $\begin{array}{l}\text { Uece- } 60 \% \\
\text { OE - } 58,6 \%\end{array}$ & $\begin{array}{l}\text { Uece- } 71,9 \% \\
\text { OE - } 69,7 \%\end{array}$ \\
\hline Insatisfeito com comportamento sexual & - & $\begin{array}{l}\text { Uece }-10 \% \\
\text { OE }-13,8 \%\end{array}$ & $\begin{array}{l}\text { Uece }-25 \% \\
\text { OE }-15 \%\end{array}$ & $\begin{array}{l}\text { Uece }-20 \% \\
\text { OE }-17,3 \%\end{array}$ & $\begin{array}{l}\text { Uece }-25 \% \\
\text { OE }-36,4 \%\end{array}$ \\
\hline
\end{tabular}

Uece $=$ Universidade Estadual do Ceará. OE = todas as outras escolas médicas do Ceará

Referir quadro depressivo acometeu 25,6\% dos alunos da Uece no primeiro ano, o que se enquadra com dados da literatura ${ }^{20}$ e representa valor superior ao da população geral, que foi de até $12 \%{ }^{21}$. A presença de problemas pessoais ou familiares tornou-se maior no último ano, com 58,6\% dos alunos da Uece referindo. A adaptação à cidade em que vive se mostrou dinâmica, com $15,5 \%$ dos alunos de outras escolas, contemplando as sediadas no interior do Estado, referindo não estarem adaptados no último ano. Quase metade dos alunos de $\mathrm{OE}$ passou a consumir álcool após ingressar na faculdade, sendo que 69,7\% consomem álcool no último ano. O consumo de drogas psicoativas mostrou dois picos na Uece: no terceiro e no sexto anos, fato repetido nas $\mathrm{OE}$. 
A privação de lazer foi praticamente homogênea ao longo dos anos, sendo mais prevalente no segundo e terceiro anos de formação. A insatisfação com o comportamento sexual foi crescente: de $13,8 \%$ a $36,4 \%$ nas OE e de $10 \%$ a $25 \%$ na Uece.

A Tabela 2 aborda os elementos acadêmicos. A angústia com as aulas práticas de Anatomia foi mais prevalente na Uece $(32,5 \%)$, o que se dá talvez pela presença de duas disciplinas de Anatomia, sendo a última destinada ao estudo da dissecação ${ }^{15}$. A vontade de desistir do curso no primeiro ano foi de $32,5 \%$ na Uece contra $24,6 \%$ nas OE. O pensamento de desistir do curso teve pico na Uece $(6,7 \%)$ no primeiro ano de internato. Já nas $\mathrm{OE}$, o valor máximo está no sexto ano. Todos os alunos da Uece e $94 \%$ das OE consideram o internato como o período mais exigente em comparação aos demais. No entanto, o último ano é pontuado por apenas $46,9 \%$ dos alunos da Uece como mais exigente que o quinto ano, o que se con- de $\mathrm{OE}$ no quarto ano. $\mathrm{O}$ temor de não ser um bom médico foi maior no último ano, atingindo $87,1 \%$ dos alunos da Uece. Esse temor contrasta com a sensação de a escola estar formando o médico que o aluno deseja ser: na Uece, houve aumento de 5 pontos percentuais do quarto ao sexto ano, enquanto decresceu também 5 pontos nas OE.

'O suporte psicopedagógico é mostrado na Tabela 3, em que $25 \%$ dos alunos da Uece procuraram ajuda psicológica no sexto ano, contra $18,2 \%$ dos alunos das demais escolas. Analisando a prevalência de alunos suspeitos de portar Transtornos Mentais Leves (TML), vê-se que, no quinto ano, 53,3\% dos alunos da Uece eram suspeitos de portar TML, mas apenas $20 \%$ procuraram ajuda. Nas OE, 48,5\% foram suspeitos, mas apenas $18,2 \%$ procuraram ajuda. No quinto ano, $76 \%$ dos alunos da Uece acharam precisar de ajuda psicológica, quando apenas $20 \%$ procuraram de fato. A oferta de apoio psicopeda-

TABELA 2

Distribuição de prevalências de acordo com o ano acadêmico estudado. Aspectos acadêmicos

\begin{tabular}{|c|c|c|c|c|c|}
\hline Variável & $2^{\circ}$ ano & $3^{\circ}$ ano & $4^{\circ}$ ano & $5^{\circ}$ ano & $6^{\circ}$ ano \\
\hline Angústia nas aulas práticas de Anatomia & $\begin{array}{l}\text { Uece - } 32,5 \% \\
\text { OE- } 10,8 \%\end{array}$ & - & - & - & - \\
\hline Pensou em desistir do curso no primeiro ano & $\begin{array}{l}\text { Uece }-32,5 \% \\
\text { OE }-24,6 \%\end{array}$ & - & - & - & - \\
\hline Pensa em desistir & - & $\begin{array}{l}\text { Uece }-5 \% \\
\text { OE }-3 \%\end{array}$ & $\begin{array}{l}\text { Uece }-2,5 \% \\
\mathrm{OE}-8,3 \%\end{array}$ & $\begin{array}{l}\text { Uece }-6,7 \% \\
\text { OE }-0 \%\end{array}$ & $\begin{array}{l}\text { Uece }-3,1 \% \\
\text { OE }-9 \%\end{array}$ \\
\hline Considera semestre mais exigente & - & $\begin{array}{l}\text { Uece }-85 \% \\
\text { OE }-81,5 \%\end{array}$ & $\begin{array}{l}\text { Uece }-67,5 \% \\
\text { OE }-53,3 \%\end{array}$ & $\begin{array}{l}\text { Uece }-100 \% \\
\text { OE }-93 \%\end{array}$ & $\begin{array}{l}\text { Uece }-46,9 \% \\
\text { OE }-42,5 \%\end{array}$ \\
\hline Mau desempenho acadêmico & $\begin{array}{l}\text { Uece }-57,7 \% \\
\text { OE }-57 \%\end{array}$ & $\begin{array}{l}\text { Uece }-27,5 \% \\
\text { OE }-35,4 \%\end{array}$ & $\begin{array}{l}\text { Uece }-35 \% \\
\text { OE }-30 \%\end{array}$ & $\begin{array}{l}\text { Uece }-6,7 \% \\
\text { OE - } 0 \%\end{array}$ & $\begin{array}{l}\text { Uece }-6,3 \% \\
\text { OE - } 12 \%\end{array}$ \\
\hline Apresenta Insônia & $\begin{array}{l}\text { Uece }-15 \% \\
\text { OE }-30,7 \%\end{array}$ & - & - & - & - \\
\hline Apresenta-se com sonolência excessiva durante as aulas & $\begin{array}{l}\text { Uece }-75 \% \\
\text { OE }-73,8 \%\end{array}$ & - & - & - & - \\
\hline Acredita haver disputa por notas & - & $\begin{array}{l}\text { Uece }-80 \% \\
\mathrm{OE}-67,7 \%\end{array}$ & $\begin{array}{l}\text { Uece }-92,5 \% \\
\text { OE }-68 \%\end{array}$ & $\begin{array}{l}\text { Uece }-46,7 \% \\
\mathrm{OE}-51,7 \%\end{array}$ & $\begin{array}{l}\text { Uece }-59,4 \% \\
\text { OE }-51,5 \%\end{array}$ \\
\hline Realiza atividades extracurriculares & $\begin{array}{l}\text { Uece }-80 \% \\
\text { OE }-63 \%\end{array}$ & $\begin{array}{l}\text { Uece }-100 \% \\
\text { OE }-73,8 \%\end{array}$ & $\begin{array}{l}\text { Uece }-100 \% \\
\text { OE }-86,7 \%\end{array}$ & $\begin{array}{l}\text { Uece }-63,3 \% \\
\text { OE }-72,4 \%\end{array}$ & $\begin{array}{l}\text { Uece }-37,5 \% \\
\text { OE }-36,4 \%\end{array}$ \\
\hline Teme não ser um bom médico & - & $\begin{array}{l}\text { Uece }-80 \% \\
\mathrm{OE}-66,1 \%\end{array}$ & $\begin{array}{l}\text { Uece }-77,5 \% \\
\text { OE }-61,7 \%\end{array}$ & $\begin{array}{l}\text { Uece }-83,3 \% \\
\text { OE }-51,7 \%\end{array}$ & $\begin{array}{l}\text { Uece }-87,1 \% \\
\text { OE }-69,7 \%\end{array}$ \\
\hline Acredita que a escola forma o médico que deseja ser & - & - & $\begin{array}{l}\text { Uece }-57,5 \% \\
\text { OE }-71,7 \%\end{array}$ & - & $\begin{array}{l}\text { Uece }-62,5 \% \\
\text { OE }-66,7 \%\end{array}$ \\
\hline
\end{tabular}

firma na literatura ${ }^{22}$. O mau desempenho acadêmico foi maior no segundo ano, atingindo os menores valores no quinto ano. No terceiro e quarto anos, todos os alunos da Uece realizavam atividades extracurriculares, sendo o pico de $86,7 \%$ de alunos gógico pela escola foi decrescente nos dois grupos, atingindo o menor valor nos últimos dois anos, que são o período de maior prevalência de suspeitos de portar TML e quando os alunos mais procuram ajuda. 
Tabela 3

Distribuição de prevalências de acordo com o ano acadêmico estudado. Aspectos ligados à assistência psicológica e aos grupos suspeitos de portar TML

\begin{tabular}{|c|c|c|c|c|c|}
\hline Variável & $2^{o}$ ano & $3^{\circ}$ ano & $4^{o}$ ano & $5^{\circ}$ ano & $6^{\circ}$ ano \\
\hline Procurou auxílio psicológico & - & - & $\begin{array}{l}\text { Uece }-20 \% \\
\text { OE }-13,3 \%\end{array}$ & $\begin{array}{l}\text { Uece }-20 \% \\
\text { OE }-20,7 \%\end{array}$ & $\begin{array}{l}\text { Uece }-25 \% \\
\text { OE }-18,2 \%\end{array}$ \\
\hline Não procurou, mas achou precisar desse auxílio & - & - & $\begin{array}{l}\text { Uece }-46,9 \% \\
\text { OE }-41,5 \%\end{array}$ & $\begin{array}{l}\text { Uece }-76 \% \\
\text { OE }-52,2 \%\end{array}$ & $\begin{array}{l}\text { Uece }-61,5 \% \\
\text { OE }-54,5 \%\end{array}$ \\
\hline Faculdade oferece auxílio psicológico & - & - & $\begin{array}{l}\text { Uece }-12,5 \% \\
\mathrm{OE}-8,3 \%\end{array}$ & $\begin{array}{l}\text { Uece }-10 \% \\
\text { OE }-3,4 \%\end{array}$ & $\begin{array}{l}\text { Uece }-6,25 \% \\
\text { OE }-0 \%\end{array}$ \\
\hline $\begin{array}{l}\text { Acha que o hospital em que realiza a maior parte } \\
\text { do internato deve oferecer auxílio ao interno }\end{array}$ & - & - & - & $\begin{array}{l}\text { Uece }-82,1 \% \\
\mathrm{OE}-79,3 \%\end{array}$ & $\begin{array}{l}\text { Uece }-90,6 \% \\
\text { OE }-84,9 \%\end{array}$ \\
\hline Alunos suspeitos de portar TML & $\begin{array}{l}\text { Uece }-35 \% \\
\mathrm{OE}-26,15 \%\end{array}$ & $\begin{array}{l}\text { Uece }-32,5 \% \\
\text { OE }-24,6 \%\end{array}$ & $\begin{array}{l}\text { Uece }-50 \% \\
\text { OE }-35 \%\end{array}$ & $\begin{array}{l}\text { Uece }-53,3 \% \\
\text { OE }-25 \%\end{array}$ & $\begin{array}{l}\text { Uece }-31,25 \% \\
\mathrm{OE}-48,5 \%\end{array}$ \\
\hline Prevalência geral & $29,5 \%$ & $27,6 \%$ & $41,4 \%$ & $42,4 \%$ & $43,9 \%$ \\
\hline Prevalência geral por sexo & $\begin{array}{l}\text { Masculino - 51,6\% } \\
\text { Feminino - } 48,4 \%\end{array}$ & $\begin{array}{l}\text { Feminino - } 62,1 \% \\
\text { Masculino - 37,9\% }\end{array}$ & $\begin{array}{l}\text { Feminino - } 65,9 \% \\
\text { Masculino - 34,1\% }\end{array}$ & $\begin{array}{l}\text { Feminino }-56 \% \\
\text { Masculino }-44 \%\end{array}$ & $\begin{array}{l}\text { Feminino - 58,6\% } \\
\text { Masculino - } 41,4 \%\end{array}$ \\
\hline $\begin{array}{l}\text { Suspeitos de portar TML de acordo com o método } \\
\text { de ensino adotado pela escola médica }\end{array}$ & $\begin{array}{l}\text { PBL }-33,3 \% \\
\text { Tradicional - } \\
28,4 \%\end{array}$ & $\begin{array}{l}\text { PBL }-16,7 \% \\
\text { Tradicional - } \\
30,9 \%\end{array}$ & $\begin{array}{l}\text { PBL }-33,3 \% \\
\text { Tradicional }-43 \%\end{array}$ & $\begin{array}{l}\text { PBL }-30 \% \\
\text { Tradicional - } \\
42,8 \%\end{array}$ & $\begin{array}{l}\text { PBL }-54,5 \% \\
\text { Tradicional - 37\% }\end{array}$ \\
\hline
\end{tabular}

A prevalência de suspeitos de portar TML é maior em mulheres a partir do terceiro ano, sendo até 31,8 pontos percentuais de diferença (quarto ano), sendo a menor diferença no quinto ano. A prevalência de suspeitos de portar TML mostrou pico no quarto ano (53,3\%) na Uece e no sexto ano nas OE $(48,5 \%)$.

Duas escolas médicas cearenses, sediadas na capital do Estado, adotam em todos os semestres letivos antecedentes ao internato metodologias ativas baseadas no PBL - Problem Based Learning. No primeiro ano, comparando com as escolas que utilizam modelos tradicionais, houve $33,3 \%$ contra $28,4 \%$ de suspeitos, respectivamente. Do terceiro ao quinto ano, as escolas com metodologias tradicionais apresentaram prevalência superior, sendo superadas no último ano de formação pelas escolas em uso regular do PBL (Tabela 3).

A Tabela 4 retrata o estudo de regressão logística com os diferentes desfechos objetivados pelo estudo. A variável de temer não ser um bom médico aparece como significativa do terceiro ao quinto ano. No primeiro ano, depressão e insônia se mostraram significativas. No quarto ano, ter algum problema pessoal ou familiar contribuiu com o sofrimento mental dos estudantes. No sexto ano, a privação de lazer teve significado no desfecho. Apesar da ausência de significado estatístico, a realização de atividades físicas regulares, o consumo de álcool, considerar que a escola forma o médico que deseja ser e realizar curso preparatório para residência médica parecem contribuir com a atenuação do sofrimento psíquico.
TABELA 4

Modelo de regressão logística

\begin{tabular}{|c|c|c|c|}
\hline Variável & Odd ratio & Coeficiente & p-value \\
\hline \multicolumn{4}{|l|}{$\begin{array}{l}\text { Desfecho "Suspeito de portar } \\
\text { TML" }\end{array}$} \\
\hline $\begin{array}{l}\text { Angústia nas aulas práticas de } \\
\text { Anatomia }\left(2^{\circ} \text { ano }\right)\end{array}$ & 2,9453 & 1,0802 & 0,0976 \\
\hline Depressão ( $2^{\circ}$ ano) & 6,2928 & 1,8394 & $0,0031^{*}$ \\
\hline Insônia ( $2^{\circ}$ ano) & 3,4169 & 1,2287 & $0,0358^{*}$ \\
\hline $\begin{array}{l}\text { Parou de realizar atividade } \\
\text { física ( } 2^{\circ} \text { ano) }\end{array}$ & 2,4291 & 0,8875 & 0,1264 \\
\hline Privou-se de lazer ( $2^{\circ}$ ano $)$ & 3,1526 & 1,1482 & 0,0957 \\
\hline $\begin{array}{l}\text { Realiza atividade física regular } \\
\left(3^{\circ} \text { ano }\right)\end{array}$ & 0,4979 & $-0,6974$ & 0,2181 \\
\hline Consome álcool ( $3^{\circ}$ ano) & 0,4411 & $-0,8185$ & 0,1136 \\
\hline $\begin{array}{l}\text { Consome alguma droga } \\
\left.\text { psicoativa ( } 3^{\circ} \text { ano }\right)\end{array}$ & 7,9289 & 2,0705 & $0,0258^{*}$ \\
\hline $\begin{array}{l}\text { Considera atual semestre mais } \\
\text { exigente }\left(3^{\circ} \text { ano }\right)\end{array}$ & 4,1440 & 1,4217 & 0,0926 \\
\hline $\begin{array}{l}\text { Teme não ser bom médico } \\
\left(3^{\circ} \text { ano }\right)\end{array}$ & 14,3350 & 2,6627 & $0,0034^{*}$ \\
\hline $\begin{array}{l}\text { Buscou auxílio psicológico ( } 4^{\circ} \\
\text { ano) }\end{array}$ & 4,0917 & 1,4090 & $0,0348^{*}$ \\
\hline $\begin{array}{l}\text { Escola forma o médico que } \\
\left.\text { deseja ser ( } 4^{\circ} \text { ano }\right)\end{array}$ & 0,5756 & $-0,5523$ & 0,2519 \\
\hline
\end{tabular}




\begin{tabular}{|c|c|c|c|}
\hline Problema familiar ( $4^{\circ}$ ano) & 3,2062 & 1,1651 & $0,0257^{*}$ \\
\hline $\begin{array}{l}\text { Teme não ser bom médico } \\
\left(4^{\circ} \text { ano }\right)\end{array}$ & 3,4929 & 1,2507 & $0,0243^{*}$ \\
\hline $\begin{array}{l}\text { Dificuldade com novas } \\
\text { amizades (5a ano) }\end{array}$ & 33,7909 & 3,5202 & $0,0057^{*}$ \\
\hline $\begin{array}{l}\text { Realiza curso preparatório para } \\
\text { residência ( } 5^{\circ} \text { ano) }\end{array}$ & 0,5418 & $-0,6129$ & 0,3908 \\
\hline Privou-se de lazer ( $5^{\circ}$ ano) & 4,3067 & 1,4602 & 0,0541 \\
\hline $\begin{array}{l}\text { Teme não ser bom médico } \\
\text { (5o ano) }\end{array}$ & 6,5228 & 1,8753 & $0,0310^{*}$ \\
\hline $\begin{array}{l}\text { Buscou auxílio psicológico } \\
\text { (5aa } \text { ano })\end{array}$ & 5,6803 & 1,7370 & 0,0399 \\
\hline $\begin{array}{l}\text { Buscou auxílio psicológico } \\
\left(6^{\circ} \text { ano }\right)\end{array}$ & 4,3129 & 1,4616 & 0,2751 \\
\hline $\begin{array}{l}\text { Consome alguma droga } \\
\text { psicoativa ( } 6^{-} \text {ano) }\end{array}$ & 8,1140 & 2,0936 & 0,1311 \\
\hline $\begin{array}{l}\text { Acha que precisou de auxílio } \\
\left.\text { psicológico ( } 6^{\circ} \text { ano }\right)\end{array}$ & 2,9171 & 1,0706 & 0,1192 \\
\hline Privou-se de lazer (6º ano) & 6,0121 & 1,7938 & $0,0387^{*}$ \\
\hline \multicolumn{4}{|l|}{$\begin{array}{l}\text { Desfecho "Mau desempenho } \\
\text { acadêmico" }\end{array}$} \\
\hline $\begin{array}{l}\text { Dificuldade com novas } \\
\left.\text { amizades ( } 2^{\circ} \text { ano }\right)\end{array}$ & $\underline{8,9084}$ & 2,1870 & $\underline{0,0007^{*}}$ \\
\hline Privou-se de lazer ( $3^{\circ}$ ano) & $\underline{0,1923}$ & $-3,0901$ & $\underline{0,0020^{*}}$ \\
\hline $\begin{array}{l}\text { Considerar o semestre atual } \\
\text { mais exigente ( } 3^{\circ} \text { ano) }\end{array}$ & $\underline{6,8237}$ & 2,1061 & $\underline{0,0352}$ \\
\hline \multicolumn{4}{|l|}{$\begin{array}{l}\text { Desfecho "Pensar em desistir } \\
\text { do curso" }\end{array}$} \\
\hline $\begin{array}{l}\text { Adaptado à cidade em que vive } \\
\left(3^{\circ} \text { ano }\right)\end{array}$ & $\underline{0,0562}$ & $-2,8793$ & $\underline{0,0156^{*}}$ \\
\hline \multicolumn{4}{|l|}{$\begin{array}{l}\text { Desfecho “Buscou ajuda } \\
\text { psicológica" }\end{array}$} \\
\hline $\begin{array}{l}\text { Curso preparatório para } \\
\text { residência médica ( } 6^{\circ} \text { ano) }\end{array}$ & $\underline{5,1789}$ & 1,6446 & $\underline{0,0172^{*}}$ \\
\hline $\begin{array}{l}\text { Problema pessoal ou familiar } \\
\left(5^{\circ} \text { ano }\right)\end{array}$ & $\underline{10,1897}$ & 2,3214 & $\underline{0,0064^{*}}$ \\
\hline $\begin{array}{l}\text { Adaptado à cidade em que vive } \\
\left(4^{\circ} \text { ano }\right)\end{array}$ & $\underline{0,0116}$ & $-4,4533$ & $\underline{0,0094^{*}}$ \\
\hline $\begin{array}{l}\text { Problema pessoal ou familiar } \\
\left.\text { ( } 4^{\circ} \text { ano }\right)\end{array}$ & $\underline{4,8172}$ & 1,5722 & $\underline{0,0263^{*}}$ \\
\hline
\end{tabular}

* Variáveis com relevância estatística com p-value $<0,05$. IC = 95\%.

Sobre o mau desempenho acadêmico, a Tabela 4 mostra que a privação de lazer no terceiro ano pode melhorar o desempenho dos alunos, diferente de considerar o semestre mais exigente ou ter dificuldade com novas amizades. Estar adaptado à cidade em que vive parece proteger contra o pensamen- to de desistir do curso e de buscar ajuda psicológica (Tabela 4). Realizar curso preparatório para residência e apresentar problema pessoal ou familiar motivam o estudante a procurar ajuda.

A Tabela 5 mostra a prevalência das variáveis entre os alunos suspeitos de portar TML nos dois grupos. Perceber mudança de humor no primeiro ano atingiu $83,9 \%$ dos suspeitos. O consumo de droga psicoativa só esteve em 11,5\% dos suspeitos no último ano (prevalência geral de suspeitos de $43,9 \%$ ). Valores expressivos colocam a ausência de atividade física regular, considerar o semestre mais exigente e temer não ser um bom médico como elementos importantes no sofrimento. Entre os suspeitos, do quarto ao sexto ano, mais de $70 \%$ consideraram precisar de ajuda psicológica, mas não a buscaram efetivamente; esse valor torna-se mais notável no quinto ano, quando $94,4 \%$ dos suspeitos não procuraram ajuda. Em todos os suspeitos não houve conhecimento de que a escola oferecesse qualquer tipo de suporte psicopedagógico no quinto e sexto anos.

\section{TABELA 5}

Prevalências gerais que envolvem alunos de todas as escolas médicas do Ceará. Prevalências das variáveis entre alunos suspeitos de portar Transtornos Mentais Leves

\begin{tabular}{|c|c|c|c|c|c|}
\hline Variável & $2^{\circ}$ ano & $3^{\circ}$ ano & $4^{\circ}$ ano & $5^{\circ}$ ano & $6^{\circ}$ ano \\
\hline ngústia com a Anatomia & $3 \%$ & - & - & - & - \\
\hline Percebeu mudança de humor & $83,9 \%$ & - & - & - & - \\
\hline Dificuldade com novas amizades & $12,9 \%$ & $20,7 \%$ & $14,6 \%$ & $29,3 \%$ & $26,9 \%$ \\
\hline Apresentou quadro depressivo & $41,9 \%$ & - & - & - & - \\
\hline Problema pessoal ou familiar & $58,1 \%$ & $55,2 \%$ & $43,9 \%$ & $41,7 \%$ & $65,4 \%$ \\
\hline Consome álcool & - & $37,9 \%$ & $53,7 \%$ & $65,2 \%$ & $57,7 \%$ \\
\hline Consome alguma droga psicoativa & - & $20,7 \%$ & $0 \%$ & $4,2 \%$ & $11,5 \%$ \\
\hline lizar ati & $74,2 \%$ & - & - & - & - \\
\hline Não realiza atividade física regular & $72,4 \%$ & $70,7 \%$ & $70,8 \%$ & $61,5 \%$ & $72,4 \%$ \\
\hline $\begin{array}{l}\text { Privou-se de alguma atividade de } \\
\text { lazer }\end{array}$ & $87,1 \%$ & $69 \%$ & $70,7 \%$ & $75 \%$ & $84,6 \%$ \\
\hline Insatisfeito com com & - & $24,1 \%$ & $24,4 \%$ & $20,8 \%$ & $34,6 \%$ \\
\hline Dieta para controle do peso & - & $24,5 \%$ & $26,8 \%$ & $25 \%$ & $34,6 \%$ \\
\hline $\begin{array}{l}\text { Sonolência excessiva dura } \\
\text { aulas }\end{array}$ & $80,6 \%$ & - & - & - & - \\
\hline mais exigente & - & $93,1 \%$ & $68,3 \%$ & $95,8 \%$ & $42,3 \%$ \\
\hline Mau deser & $58,1 \%$ & $44,8 \%$ & $36,6 \%$ & $8,3 \%$ & $11,5 \%$ \\
\hline Atividades complementares & $74,2 \%$ & $86,2 \%$ & $97,6 \%$ & $54,2 \%$ & $34,6 \%$ \\
\hline forma o & $\begin{array}{l}- \\
-\end{array}$ & - & $41,5 \%$ & - & $38,5 \%$ \\
\hline
\end{tabular}




\begin{tabular}{|c|c|c|c|c|c|}
\hline Teme não ser um bom médico & - & $93,1 \%$ & $85,4 \%$ & $83,2 \%$ & $84,6 \%$ \\
\hline Procurou auxílio psicológico & - & - & $29,3 \%$ & $29,2 \%$ & $30,8 \%$ \\
\hline $\begin{array}{l}\text { Não procurou, mas achou precisar } \\
\text { desse auxílio }\end{array}$ & - & - & $70 \%$ & $94,4 \%$ & $76,2 \%$ \\
\hline $\begin{array}{l}\text { Faculdade não oferece auxílio } \\
\text { psicológico }\end{array}$ & - & - & - & $100 \%$ & $100 \%$ \\
\hline $\begin{array}{l}\text { Acha que o hospital deveria } \\
\text { oferecer auxílio ao interno }\end{array}$ & - & - & - & $92,3 \%$ & - \\
\hline
\end{tabular}

\section{DISCUSSÃO}

Avaliando o primeiro ano de formação, nos dois grupos estudados, percebeu-se o impacto da transição do ensino médio à formação superior. A percepção de mudança de humor, quadro depressivo, mau desempenho acadêmico, dificuldade com novas amizades, consumo de drogas psicoativas e o pensamento de desistir do curso sugerem que o primeiro ano representa uma fase de transição permeada de incertezas e adaptações do estudante.

Em um retrato geral, é nítida a distância entre suspeitos de portar TML entre os gêneros ${ }^{23,24}$. Já na avaliação geral dos alunos suspeitos, há inclinação da literatura para três picos estressores: transição ao ciclo clínico, ano que antecede o internato (quarto ano) e o último ano, conforme dados de alguns autores $23,25,26$. A despeito do aumento progressivo da prevalência de alunos em sofrimento psíquico, ainda são débeis as iniciativas das escolas médicas e o reconhecimento do próprio adoecimento pelos alunos, o que não os motiva a buscar ajuda ${ }^{23,27}$.

Tentando-se, então, compreender a construção do subjetivismo do sofrimento mental, vê-se que a transição do modelo pedagógico expositivo clássico do ensino médio é sentida principalmente nas escolas que adotam metodologias ativas de forma regular em sua grade, enquanto o modelo tradicional de outras escolas, mais próximo do ensino médio, mostrou menor prevalência, o que se confirma pela literatura ${ }^{28}$. Essa fase de adaptação ao novo método de ensino não paternalista exige do estudante mudança de postura e muita dedicação, obrigando-o a abdicar do lazer, exercícios e convívio social. Tais perdas podem, assim, provocar dor psíquica, vivência depressiva, tristeza, frustração e desânimo ${ }^{1}$. Ressalta-se ainda que, na literatura, os estudantes do primeiro ano de Medicina se queixam da limitação importante de oportunidades para o desenvolvimento de relações pessoais ${ }^{9}$.

A percepção de angústia com as aulas de Anatomia indica que o enfrentamento da morte e sua personificação precisam ser mais bem abordadas, no caso, principalmente, na
Uece, em que o contato com o cadáver se dá durante todo o primeiro ano e quando o aluno não dispõe de disciplinas ou orientação específica sobre a compreensão da morte ${ }^{15}$. O contato com a Anatomia tem despertado sentimentos de angústia e desgosto nos estudantes, além de sentimentos que se assemelham ao estresse pós-traumático, como também pesadelos e insônia ${ }^{29}$.

A carga horária extenuante, em turno integral, além da necessidade de novos hábitos de estudo, parece ter provocado um modelo em que o aluno precisou se privar de atividades de lazer e parar de realizar atividades físicas, o que mostrou impacto significativo sobre o desfecho do sofrimento mental no último ano (Tabela 4). Além disso, a privação de lazer contribuiu para o mau desempenho acadêmico (Tabela 4), significando que o aluno precisou cercear outras atividades lúdicas para poder apresentar melhor desempenho nas avaliações. No entanto, a dificuldade com novas amizades, representando a deficiência na constituição de uma rede de ajuda mútua e de bom relacionamento social, favoreceu o mau desempenho acadêmico no primeiro ano, podendo-se compreender a importância da integração social no enfrentamento dos testes e avaliações.

A elaboração de teias sociais, considerando que o curso de Medicina exige dedicação em tempo integral do estudante, é um ponto importante, pois ajuda a estabelecer vínculos e cria reciprocidades, o que pode atenuar o sofrimento dos estudantes. No início do internato, quando o aluno passa a conviver com residentes, preceptores e alunos de outras instituições, a dificuldade com novas amizades foi maior nos alunos da Uece. No entanto, no último ano, os alunos de OE tiveram maior prevalência, o que se pode explicar pelo fato de os alunos da Uece serem em menor número, utilizarem os hospitais de internato como campo de aula prática desde o quarto semestre letivo e passarem a maior parte do internato em um único hospital, o que facilita a criação de vínculos.

O consumo de álcool parece também ter efeito sobre o adoecimento: apesar de não haver significado estatístico, o consumo de álcool parece atenuar o sofrimento mental dos estudantes, a princípio, ao passo que no último ano quase $70 \%$ dos alunos consomem bebidas alcoólicas, o que indica, pelo menos, participação em grupos ou atividades de lazer coletivas. $\mathrm{O}$ aumento progressivo do consumo de álcool ao longo do curso médico é igualmente sinalizado na literatura ${ }^{7}$. O consumo de álcool pode ser ainda analisado quando o maior pico de elevação de consumo de álcool entre os alunos estudados se deu no quarto ano, período que coincide com o também maior aumento na prevalência de alunos suspeitos de portar TML. De forma geral, o uso de drogas entre estudantes de 
Medicina tem aumentado, apesar das informações a que têm acesso, e os dados sugerem que há necessidade de alocar recursos para o tratamento, recuperação e reabilitação de alunos que já abusam ou possam ser dependentes de drogas ${ }^{7}$.

O ambiente competitivo, talvez um resquício do processo seletivo acirrado para ingressar no curso de Medicina, ocorre principalmente no quarto ano, o período que antecede o internato. É nesse ano que os alunos passam a realizar o maior número de atividades extracurriculares em busca de um currículo paralelo que não tenham obtido até o terceiro ano, currículo este previsto como necessário ao sucesso na aprovação em residências médicas. Com o início do internato, o aluno apresenta menos tempo disponível para essas atividades e, portanto, precisa desempenhá-las no ano anterior, que coincide com o período de maior aumento na prevalência de alunos suspeitos de portar TML.

A transição para o ciclo clínico é apontada como forte elemento estressor por outros autores ${ }^{27,30}$. No entanto, a pesquisa mostrou diminuição na prevalência de alunos suspeitos de portar TML nos dois grupos, o que se credita aos atuais currículos, em que as disciplinas são orientadas por módulos, e o contato com o ciclo clínico já se dá nos primeiros anos, representando pouco impacto nos anos seguintes. Na Uece, mesmo com currículo por disciplinas, o ciclo clínico parece ter atenuado o sofrimento dos estudantes, o que se justifica pela descoberta do novo, pelo contato mais próximo com a prática médica e pela realização das ideações iniciais. No entanto, no ano seguinte, quando o ciclo clínico já se mostra mais exigente, há o maior aumento no número de suspeitos de portar TML, a despeito de ter sido considerado período menos exigente que o anterior. Esse fato revela a importância das atividades extracurriculares sobre o sofrimento dos estudantes, além da ansiedade com o início do internato, em que o aluno percebe a demanda de mais habilidades práticas, passa a se questionar acerca da qualidade como futuro médico e já se prepara para os concursos de residência médica.

O consumo de drogas psicoativas contempla drogas lícitas e ilícitas, como psicofármacos de indicação médica. Nos anos de maior prevalência de suspeitos de portar TML, não houve as maiores taxas de uso dessas drogas, mas o terceiro ano se mostrou com uso aumentado, o que pode representar uma rota de escape dos estudantes ao sofrimento acumulativo dos primeiros anos de formação, conforme indica a literatura ${ }^{7}$. Outro pico no uso dessas drogas se deu no último ano, quando o aluno já tem domínio sobre a indicação de psicofármacos, tem o acúmulo de desordens mentais ao longo dos anos e já reconhece seu próprio sofrimento, o que se vê com o aumento da procura de ajuda psicológica ou a percepção de que precisou desse auxílio. Os valores, então, parecem ganhar volume com os psicofármacos dos alunos em acompanhamento médico.

O modelo de formação e a proximidade da escola com a individualidade do estudante são itens importantes. A falta de segurança do estudante com a qualidade do profissional que será aparece desde o terceiro ano, quando também apresenta seu pico. No internato, quando o aluno passa a assumir responsabilidades de um "quase médico" e tem a oportunidade de praticar o que aprendeu nos anos anteriores, há uma melhora desse temor, o que conforta mais o estudante. Assim, a sensação de que a escola forma o médico que deseja ser teve relação entre o ano anterior ao internato e no último ano. No grupo da Uece, houve melhora da segurança dos alunos com a escola no último ano, o que se pode creditar aos resultados obtidos pela Uece em sua primeira avaliação do Enade/MEC, em que a escola logrou nota máxima, sendo o êxito bastante divulgado entre os alunos ${ }^{31}$. Os resultados do ano anterior ao internato se explicam pela ausência de uma autoavaliação prática ou por terceiros do que o aluno está apto a fazer como futuro médico, o que, portanto, pode não lhe conferir segurança técnica.

A ambiência externa tem contribuição importante sobre o sofrimento dos estudantes. Não se achar adaptado à cidade em que vive e apresentar problemas pessoais ou familiares são os pontos de significância para o estudante procurar atendimento psicológico. A análise dessa ambiência indica que, a despeito da relação dos elementos ligados à formação acadêmica diretamente, o estudante parece compreender que apenas as interferências externas motivam a busca por ajuda, avolumando os problemas ligados à formação acadêmica sem o devido amparo psicológico. A participação em cursos preparatórios de residência médica contribuiu com a procura por ajuda psicológica, pois certamente esses cursos suscitam o ambiente competitivo do vestibular, expõem os estudantes a testes simulados e rankings, e apresentam conteúdos que muitas vezes não são abordados de forma eficiente nos anos anteriores, potencializando a percepção do estudante de que ele precisa de ajuda para superar a nova realidade.

No grupo da Uece, destaca-se a evolução dos alunos suspeitos de portar TML. No último ano, a prevalência foi praticamente igual à do início da coorte, com pico no primeiro ano de internato, diferentemente do encontrado nas $\mathrm{OE}$, sugerindo que no quarto e quinto anos houve uma estabilização na quantidade de suspeitos de portar TML, gerando a percepção da necessidade de meios que pudessem atenuar esse transtorno. Por isso, houve aumento na procura por atendimento psicológico, diminuição na dificuldade com novas amizades, realização de atividades físicas regulares, maior segurança 
com a qualidade dos médicos formados pela universidade e diminuição das atividades extracurriculares. Esse fenômeno pode ser explicado pela turma reduzida de alunos, pelo vínculo maior que se forma com a escola, pelas conquistas acadêmicas da universidade nos últimos anos e pelos resultados e posições profissionais dos primeiros ex-alunos, que estão em período de conclusão de residência médica ${ }^{31}$. Já nas $\mathrm{OE}$, a maior prevalência de alunos suspeitos de portar TML no último ano representa as incertezas quanto à atuação profissional, a maior privação de lazer e de problemas pessoais ou familiares, insatisfação com o comportamento sexual, o ambiente do internato como um processo de avaliação constante e formativo da imagem como futuro médico, o temor de não ser um bom médico e o convívio acumulativo com o sofrimento dos pacientes, além da rotina mais extenuante do último ano, quando se alia à realização de cursos preparatórios para residência médica, apontada como um processo que requer atenção ${ }^{32}$.

Soma-se a isso o fato de as escolas apresentarem núcleos de apoio psicopedagógico de forma ineficiente, pois, nos últimos dois anos, quando há maior prevalência de alunos suspeitos de portar TML, todos os alunos suspeitos dizem desconhecer qualquer forma de apoio vinda da escola médica. As escolas médicas devem projetar currículos não apenas para o conteúdo médico, mas também considerando o enfrentamento emocional dos alunos ao longo do processo de formação. Maior esforço deve ser feito para diminuir a competitividade e as autoexigências excessivas, por meio de grupos de discussão, mentoring e apoio psicopedagógico ${ }^{9}$. Nos últimos anos, os alunos aprimoram a percepção do sofrimento mental, mas, mesmo assim, não recebem apoio e não o buscam, quando quase $95 \%$ dos suspeitos dizem ter percebido a necessidade do apoio, mas não o terem feito de fato, o que se confirma na literatura ${ }^{5,33,34}$. No internato, também foi crescente a necessidade de apoio psicopedagógico dentro dos hospitais em que se realiza a maior parte do estágio.

\section{CONCLUSÕES}

A formação médica expõe o estudante a diversos fatores tensionais, representando uma fase de transição do Ensino Médio permeada de dúvidas e incertezas. A qualidade da escola e a segurança em uma formação médica de qualidade influenciam sobre o sofrimento psíquico, sendo a transição para o ciclo clínico um período de pouca influência, mas que representa fator estressor nos anos seguintes e se torna simultâneo ao aumento das atividades extracurriculares e à busca por um currículo paralelo, suscitando o ambiente competitivo como resquício do vestibular. No quarto ano, o período de maior prevalência de suspeitos de portar TML, também há a maior queixa de disputa por notas pelos alunos.

A progressão cronológica do curso médico aumenta a prevalência do sofrimento mental dos estudantes, sendo fortes implicadores os processos ligados à formação acadêmica. No entanto, os alunos passam a compreender melhor a própria saúde mental, recorrendo a serviços de ajuda psicológica. Porém, a grande maioria dos alunos em sofrimento psíquico não procura ajuda e, quando o faz, motiva-se essencialmente por questões pessoais ou familiares, desconsiderando os elementos acadêmicos visivelmente implicados no sofrimento.

Os currículos e os modelos de ensino precisam levar em consideração a forma como os estudantes lidam com o processo de formação em seu contexto biopsicossocial. As escolas devem tornar seus serviços de apoio psicológico mais integrados à execução dos planos de ensino, focando os dois últimos períodos acadêmicos e estendendo suas ações às unidades de cumprimento de internato. Além disso, oferecer suporte às tensões familiares e ao enfrentamento da adaptação à cidade em que o aluno vive deve fazer parte do apoio psicopedagógico.

Assim, as escolas devem agir de forma ativa na oferta de apoio psicopedagógico aos futuros médicos, reconhecendo os elementos naturalmente estressores, avaliando conteúdos que diminuam o impacto de outras disciplinas, integrando-se ao enfrentamento dos casos pessoais e familiares e aprimorando seus métodos de ensino de forma a aperfeiçoar as transições por diferentes períodos e ciclos. Devem ainda estimular a realização de atividades extracurriculares de forma ponderada e afinada com os objetivos da formação, oferecendo segurança e qualidade para que o aluno sinta que será um bom médico, sem desprestigiar as atividades de lazer e suas diversas teias sociais.

\section{LIMITAÇÕES DO ESTUDO}

O estudo poderia ter utilizado outros instrumentos complementares para avaliação do processo de sofrimento ou adoecimento mental, avaliando outros elementos do processo. A amostra do grupo de comparação poderia ter contemplado mais alunos, o que tornaria os dados mais fidedignos. A coleta de dados, apesar de não ter sido objetivo da pesquisa, poderia ter iniciado a avaliação desde o primeiro ano da graduação, contemplando a carga de alterações psíquicas advindas do processo seletivo de ingresso no curso de Medicina.

\section{REFERÊNCIAS}

1. Millan LR, DeMarco OLN, Rossi E, Arruda PCV, org. O Universo psicológico do futuro médico, vocação, vicissitudes e perspectivas. São Paulo: Casa do Psicólogo; 1999. 
2. Bellodi PL. Retaguarda Emocional para o Aluno de Medicina da Santa Casa de São Paulo (Repam): Realizações e Reflexões. Revista Brasileira De Educação Médica 2007; 31(1): 5-14.

3. Quintana AM, Rodrigues AT; Arpini DM; Bassi LA, Cecim PS, Santos MS. A angústia na formação do estudante de medicina. Revista Brasileira de Educação Médica 2008; 32(1): 7 - 14 .

4. Benevides-Pereira AMT, Gonçalves MB. Transtornos emocionais e a formação em Medicina: um estudo longitudinal. Revista Brasileira De Educação Médica 2009; 33(1):10 - 23

5. Melo-Carrillo A, Oudenhove LV, Lopez-Avila A. Depressive symptoms among Mexican medical students: High prevalence and the effect of a group psychoeducation intervention. Journal Of Affective Disorders 2012; 136: 10981103.

6. Costa EF, Santana YG, Santos ATRA, Martins LAN, Melo EV, Andrade TM . Sintomas depressivos entre internos de medicina em uma universidade pública brasileira. Rev Assoc Med Bras 2012; 58(1): 53-59.

7. Oliveira, LG, Barroso LP, Wagner GA, Ponce JC; Malbergier A, Stempliuk VA, Andrade AG. Drug consumption among medical students in São Paulo, Brazil: influences of gender and academic year. Rev Bras Psiquiatr. 2009; 31(3): 227-239.

8. Frank, Erica; Hedgecock, Joan; Elon, Lisa K. Personal health promotion at US medical schools: a quantitative study and qualitative description of deans. BMC Medical Education [on line]. 2004; 29(4) [capturado 25 maio 2013]. Disponível em: http://www.biomedcentral.com/1472$6920 / 4 / 29$

9. Tempski P, Bellodi PL, Paro HB, Enns SC, Martins MA, Schraiber LB. What do medical students think about their quality of life? A qualitative study. BMC Medical Education [on line]. 2012; 106(12). [capturado 19 maio 2013]; 1-8. Disponível em: http://www.biomedcentral. com/1472-6920/12/106

10. Trindade LMDF, Vieira MJ. Medical School: motivations and expectations of incoming students. Revista Brasileira de Educação Médica 2009; 33(4): 542-554.

11. Loureiro EMF, McIntyre TM; Mota-Cardoso R; Ferreira MA. Inventário de Fontes de Estresse Acadêmico no Curso de Medicina (IFSAM). Revista Brasileira De Educação Médica 2009; 33(2):191-197.

12. Dyrbye LN, Thomas MR, Shanafelt T. Systematic Review of Depression, Anxiety, and Other Indicators of Psychological Distress Among U.S. and Canadian Medical Students.Academic Medicine 2006; 81(4): 354-373.
13. Shah M, Hasan S, Malik S, Sreeramareddy CT. Perceived Stress, Sources and Severity of Stress among medical undergraduates in a Pakistani Medical School. Bmc Medical Education [on line]. 2010; 10(2) [capturado 08 jun. 2013]; 1-8. Disponível em: http://www.biomedcentral. com/1472-6920/10/2.

14. Mari JJ, Williams PA. A validity study of a Psychiatric Screening Questionnaire (SRQ 20) in primary care in city of Sao Paulo. Br J Psychiatry 1986;148:23-26.

15. Universidade Estadual do Ceará. Projeto Político Pedagógico do Curso de Medicina. Fortaleza: Pró-Reitoria de Graduação; 2012.

16. Comissão de Reforma Curricular. Projeto Pedagógico: Currículo do Curso de Medicina. Fortaleza: Imprensa Universitária, 2001. 72 p. [on line] [capturado 20 abr. 2013] Disponível em: http:/ / www.medcariri.ufc.br/cont/serie \%20 academica.pdf.

17. Centro Universitário Christus. Curso de Medicina [on line] [capturado 22 abr. 2013]. Disponível em: http:/ / www. fchristus.com.br/index.php?option=com_content\&view= article\&id $=73 \&$ Itemid $=121$

18. Universidade de Fortaleza. Curso de Medicina [on line] [capturado 05 maio 2013]. Disponível em: http:/ / www. unifor.br/index.php?option=com_content\&view=article\& $\mathrm{id}=341$ \&Itemid $=393$

19. Faculdade de Medicina Estácio de Juazeiro do Norte. Regimento Acadêmico do Curso de Medicina. [on line] [capturado 05 maio 2013.] Disponível em: http:/ / portal.estacio. br/media/1610606/cond_oferta_curso_medicina.pdf.

20. Yiu V. Supporting the well-being of medical students: Commentary. Cmaj 2005; 172(7):889-890.

21. Barbosa GA, org. A Saúde dos Médicos no Brasil. Brasília: Conselho Federal de Medicina; 2007 (Relatório)

22. Meyer C, Guimarães ACA; Machado Z; Parcias SR. Qualidade de Vida e Estresse Ocupacional em Estudantes de Medicina. Revista Brasileira De Educação Médica 2012; 36(4):489-498.

23. Cunha MAB, Neves AAF, Moreira ME, Hehn FJ, Lopes TP, Ribeiro CCF, Watanabe APF. Transtornos psiquiátricos menores e procura por cuidados em estudantes de Medicina. Revista Brasileira De Educação Médica 2009; 33(3): 321-328.

24. Fiedler PT. Avaliação da qualidade de vida do estudante de medicina e da influência exercida pela formação acadêmica. São Paulo, 2008. Doutorado [Tese] - Departamento de Medicina Preventiva, Universidade de São Paulo, Disponível em: <http://www.abem-educmed.org.br/ pdf_tese/tese_patricia_tempski.pdf $>$. Acesso em: 04 maio 2013. 
25. Aguiar SM. Prevalência de sintomas de estresse e de depressão nos estudantes de medicina e de odontologia. Fortaleza; 2007. Mestrado [Dissertação] - Curso de Mestrado em Saúde Pública, Universidade de Fortaleza.

26. Guthrie EA, Black D, Bagalkote H, Shaw C, Campbell M, Creed F. Psychological stress and burnout in medical students: a five-year prospective longitudinal study. Journal Of The Royal Society Of Medicine 1998;91(1): 237-243.

27. Roberts, LW, Hardee, JT, Franchini, G, Stidley, C, Siegler, M. Medical students as patients: a pilot study of their health care needs, practices and concerns. Acad Med. 1996;71: 1225-32.

28. Lewis AD, Menezes DAB, McDermott HE, Hibbert LJ, Brennan S, Ross EE, Jones LA. A comparison of course-related stressors in undergraduate problem-based learning (PBL) versus non-PBL medical programmes. BMC Medical Education [on line]. 2009;60(6). [capturado 05 maio 2013]. 1-8. Disponível em: http://www.biomedcentral. com/1472-6920/9/60

29. Weurlander M, Scheja M, Wernerson A. Emotionally challenging learning situations: medical students' experiences of autopsies. International Journal of Medical Education 2012; 3(1): 63-70.

30. Whyte R. Medical students' experience of personal loss: incidence and implications. Bmc Medical Education [on line]. 2013. 36(13) [capturado 28 maio 2013];1-7. Disponível em: http:/ / www.biomedcentral.com/1472-6920/13/36

31. Andrade JBC, Silva, MGC. Medicina na UECE: A década que levou ao máximo. Fortaleza: EdUECE; 2013.

32. Gavioli MA. Formando grupos no internato: critérios de escolha, satisfação e sofrimento psíquico. Revista Brasileira De Educação Médica 2009;33(1): 4-9.

33. Grotmol KS, Gude T, Moum T, Vaglum P, Tyssen R. Risk factors at medical school for later severe depression: A 15year longitudinal,nationwide study(NORDOC).Journal Of Affective Disorders 2013; 146: 106-111.
34. Aquino MT. Prevalência de transtornos mentais entre estudantes de medicina da Universidade Federal de Minas Gerais. Belo Horizonte; 2012. Mestrado [Dissertação] - Curso de Programa de Pós-graduação em Ciências da Saúde, Universidade Federal de Minas Gerais.

\section{CONTRIBUIÇÃO DOS AUTORES}

João Brainer Clares de Andrade - revisão de literatura, desenho do método, criação dos formulários e redação do artigo. José Jackson Coelho Sampaio - desenho do método, supervisão da coleta de dados e redação final do artigo. Lara Maciel de Farias - coleta de dados e revisão de literatura. Lucas da Ponte Melo - coleta de dados, revisão de literatura e suporte à coleta de dados virtual. Dalmy Pinheiro de Sousa - coleta de dados, revisão de literatura e criação e gestão do banco de dados. Ana Luisa Barbosa de Mendonça - coleta de dados e revisão de literatura. Francisco Felinto Aguiar de Moura Filho - coleta de dados, revisão de literatura e formatação do artigo. Ingrid Sorensen Marinho Cidrão - coleta de dados, revisão de literatura e interpretação dos dados.

\section{CONFLITO DE INTERESSES}

Os autores declaram não haver qualquer conflito de interesse. $\mathrm{O}$ financiamento da pesquisa foi realizado pelos autores. $\mathrm{O}$ autor principal é bolsista do Conselho Nacional de Pesquisa.

\section{ENDEREÇO PARA CORRESPONDÊNCIA}

João Brainer Clares de Andrade

Rua República do Líbano, 300 - apto 401

Beta - Fortaleza

CEP 60160-140 - CE

E-mail: joaobrainer@gmail.com 